\title{
A GM $(1,1)$ Markov Chain-Based Aeroengine Performance Degradation Forecast Approach Using Exhaust Gas Temperature
}

\author{
Ning-bo Zhao, Jia-long Yang, Shu-ying Li, and Yue-wu Sun \\ College of Power and Energy Engineering, Harbin Engineering University, Harbin 150001, China \\ Correspondence should be addressed to Ning-bo Zhao; zhaoningbo314@126.com
}

Received 15 December 2013; Revised 9 March 2014; Accepted 16 March 2014; Published 6 April 2014

Academic Editor: Qingsong Xu

Copyright ( 2014 Ning-bo Zhao et al. This is an open access article distributed under the Creative Commons Attribution License, which permits unrestricted use, distribution, and reproduction in any medium, provided the original work is properly cited.

\begin{abstract}
Performance degradation forecast technology for quantitatively assessing degradation states of aeroengine using exhaust gas temperature is an important technology in the aeroengine health management. In this paper, a GM $(1,1)$ Markov chain-based approach is introduced to forecast exhaust gas temperature by taking the advantages of GM $(1,1)$ model in time series and the advantages of Markov chain model in dealing with highly nonlinear and stochastic data caused by uncertain factors. In this approach, firstly, the GM $(1,1)$ model is used to forecast the trend by using limited data samples. Then, Markov chain model is integrated into GM $(1,1)$ model in order to enhance the forecast performance, which can solve the influence of random fluctuation data on forecasting accuracy and achieving an accurate estimate of the nonlinear forecast. As an example, the historical monitoring data of exhaust gas temperature from CFM56 aeroengine of China Southern is used to verify the forecast performance of the GM $(1,1)$ Markov chain model. The results show that the GM $(1,1)$ Markov chain model is able to forecast exhaust gas temperature accurately, which can effectively reflect the random fluctuation characteristics of exhaust gas temperature changes over time.
\end{abstract}

\section{Introduction}

Prognostic and health management for aeroengine are the main concerns for many researchers and users in order to provide more useful information for the safe operation $[1,2]$. Performance degradation forecast technology for assessing degradation states quantitatively based on gas path performance parameters is one of the most important technologies, which can improve the safety, reliability, and maintenance of aeroengine $[3,4]$. Therefore, how to improve the forecast precision by the advanced condition monitoring techniques to extract hidden, unknown, and the useful information from large amounts of monitoring data is emphasized on in the study of performance degradation forecast.

In the past half-century, different methods have been developed to analyze aeroengine gas path performance parameters for performance degradation $[5,6]$, fault diagnosis [7], remaining service life [8-10], and reliability [11]. Li developed a novel adaptive gas path analysis (adaptive GPA) approach to estimate actual engine performance and gas path component health status by using gas path measurements [5]. Chen established the Artificial Neural Network (ANN) model of aeroengine performance trend forecasting by using the strong nonlinear mapping ability of ANN and the phase space reconstruction theory [6]. Jiang took complex engine gas path system as a grey system and evaluated the samples utilizing the grey relationship degree theory to achieve the gas path fault diagnosis of aeroengine [7]. Wu et al. proposed Support Vector Machines (SVM) to predict the residual life of aeroengine based on the data of the actual gas path parameters monitoring information and failure event report from the aeroengine [8]. Ren and Zuo developed a residual life prediction model based on Bayesian updating methods and particle swarm optimization with immunity algorithms through analyzing the performance degradation process of aeroengine [9]. On the basis that the gas path performance parameters of aeroengine were analyzed, the aeroengine residual life prediction method based on performance deterioration pattern was proposed by Fu et al. [10]. Wang and Jiang provided a performance reliability prediction method based on Support Vector Machines (SVM) for aeroengine by using condition monitoring information [11].

Several approaches have been introduced to forecast the gas path performance parameters of aeroengine. Based on 
the research of aeroengine performance parameters relativity, with the condition of small samples and variables with multiple correlations, Shi et al. proposed a partial leastsquares regression method to build short time forecasting model of aeroengine performance parameter under the condition of small samples [12]. In order to improve the forecasting accuracy of aeroengine performance parameters, $\mathrm{Li}$ et al. decomposed the original sequence by using wavelet transform and forecasted the subsequences in different frequency bands by using Auto Regressive Moving Average (ARMA) or Auto Regressive Integrated Moving Average (ARIMA) [13]. Zhong et al. adopted the process neural networks to predict aeroengine performance parameters [1416]. Zhang and Wang adopted Support Vector Machine (SVM) regression approach to monitor an aeroengine health and condition by building monitoring models of main aeroengine performance parameters [17]. In order to predict the change tendency of aeroengine performance parameters effectively, a novel exhaust gas temperature (EGT) prediction method named process support vector machine (PSVM) was proposed by Fu and Zhong [18]. Ilbas and Turkmen dealt with the estimation of exhaust gas temperature (EGT) of a CFM56$7 \mathrm{~B}$ turbofan engine using Artificial Neural Network (ANN) at two different power settings, maximum continuous and takeoff [19].

From the literature described above, statistical and artificial intelligence based approaches are the two main techniques. Auto Regressive (AR), Moving Average (MA), Auto Regressive Moving Average (ARMA), and Auto Regressive Integrated Moving Average (ARIMA) can be mentioned as statistical models, while Artificial Neural Network (ANN) and Support Vector Machines (SVM) have been most widely used as artificial intelligence approaches. The essences of the above approaches are establishing the appropriate time series model by analyzing historical data. The modeling processes of the statistical based approaches are relatively simple $[12,13]$. However, these approaches for accurately forecasting trends depend on the law of the distribution of historical data as well as large amounts of observed data. ANN-based approaches eliminate the limitations of traditional regression methods and accurately establish mapping between input and output variables $[19,20]$. It can approximate an arbitrary nonlinear function with satisfactory precision. A great deal of training data and relatively long training period for robust generalization can ensure the success of ANN-based approaches. Although the support vector machines-based approach has many special advantages that can resolve problems, such as the small-sample set, nonlinear, and high dimensions. The kernel functions and penalty factor are difficult to determine, which can also influence the forecast accuracy [17, 18]. Conventional methods of accurate forecast time series trends depend on the sufficiency and completeness of the information obtained.

In practical applications, it is difficult to obtain the complete information because of many reasons. Besides, the aeroengine gas path performance parameters are often highly nonlinear, stochastic, and nonstationary. Therefore, not only the conventional statistical models are not as accurate as the artificial neural network-based approaches for aeroengine gas path performance parameters trend forecast problems, but the traditional methods may also be too complex to be used in forecasting future values of time series.

Grey system theory, proposed by Deng in 1982 [21], is a mathematical analysis of systems with incomplete information and discrete data. As a simple forecast model, GM (1, 1) model has been widely and successfully applied to various systems such as economic and industrial and so forth [22]. In aeroengine, Wu introduced a wear fault forecast model based on grey system theory for aeroengine [23]. The results showed that compared with the traditional prediction methods of time sequence $\operatorname{AR}(n)$ model, the forecast method of the GM $(1,1)$ model has the advantages of higher precision of forecast and lower sensitivity to the unequal intervals among the original data sequences for the modeling. However, the forecast accuracy of GM $(1,1)$ model is unsatisfactory when original data shows great randomness because GM $(1,1)$ model is only a just order single variable grey model [24].

The goal of the paper is to introduce the time series forecast based on grey system theory to the forecast modeling of aeroengine gas path performance parameters. Firstly, a type of time series forecast method based on GM $(1,1)$ model is introduced for aeroengine exhaust gas temperature (EGT). This method can effectively solve the trend forecast problems of EGT under incomplete information and discrete small sample data. Then, Markov chain model is integrated into GM $(1,1)$ model in order to enhance the forecast performance, which can solve the influence of random fluctuation data on forecast accuracy and achieve an accurate estimate of the nonlinear EGT. A real case of aeroengine EGT from CFM56 aeroengine of China Southern is used to test the capability of the proposed improved model.

The rest of this paper is organized as follows. In Section 2, the degradation signature of aeroengine is analyzed. Section 3 briefly describes the modeling methodologies of aeroengine based on GM $(1,1)$ model. The hybrid model which combines Markov chain model with GM $(1,1)$ model is discussed in Section 4 . The application and discussion are illustrated and detailed in Section 5. Finally, some conclusions are presented in Section 6.

\section{Aeroengine Degradation Signatures and Problem Description}

The performance of an aeroengine will deteriorate over the time due to different gas path component degradations such as fouling, erosion, corrosion, and foreign object damage [25]. There are many gas path performance monitoring parameters for civil aeroengine. Due to the fact that performance degradation modes of different parts are different with the increase of aeroengine service time, it is very important to select the appropriate measured parameters that can reflect the performance degradation of aeroengine to realize aeroengine performance degradation forecast.

Outlet temperature of combustor chamber is the most important performance parameter for aeroengine. Not only does it affect the overall performance of the engine, but also it directly determines the ultimate strength of turbine blade. 


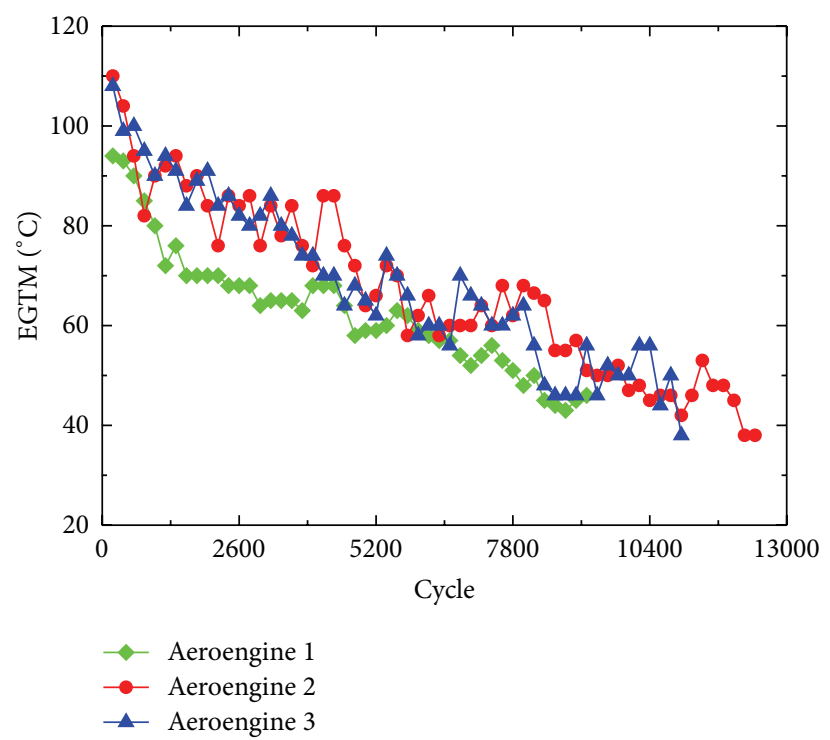

(a)

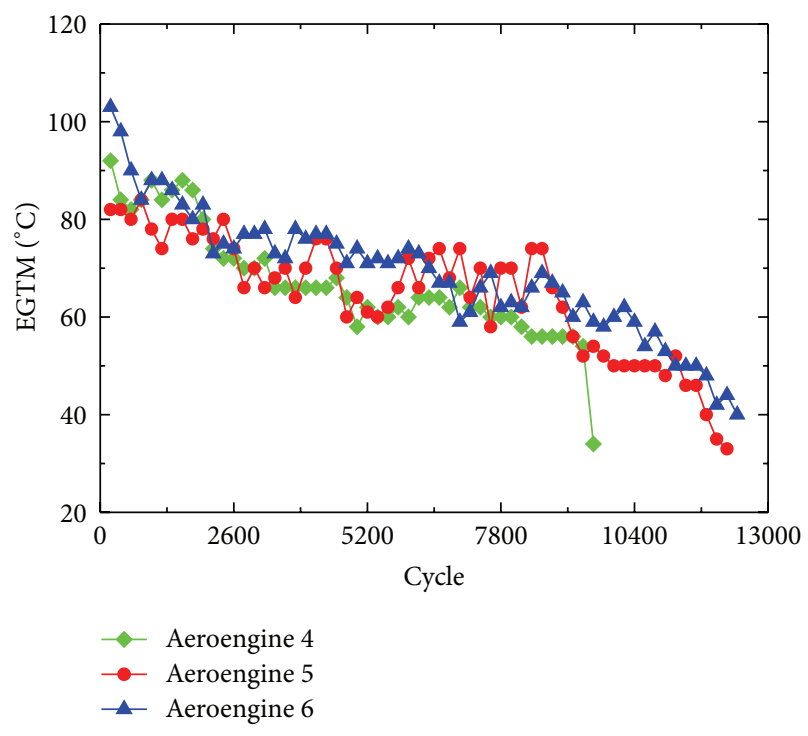

(b)

FIGURE 1: EGTM sequence and trend analysis for CFM56 aeroengine [31].

For example, the creep life of hot channel components can reduce the order of magnitude when outlet temperature of combustor chamber increases $50^{\circ} \mathrm{C}$ [26], which may cause major fault and incur great maintenance costs. However, outlet temperature of combustor chamber is usually too high to be measured with available instrumentation and temperature distribution is extremely uneven. According to the well-defined Brayton thermodynamic cycle, there is a consistent relationship between outlet temperature of combustor chamber and exhaust gas temperature (EGT). Thus, EGT, as a measured parameter, is often used for engine control, condition monitoring, fault diagnosis, and maintenance decisions. When other conditions remain the same, the higher the EGT is, the more serious the performance degradation of aeroengine is.

Considering the gas path performance monitoring parameters, the multiple linear regression models for the relationship between EGT and other parameters were established by Song et al. [27]. The results showed that there were a strong linear correlation between the performances parameters, such as low turbine outlet pressure, high rotational speed, high pressure compressor outlet temperature, low rotational speed, and high pressure compressor outlet pressure can be reflected through the change of EGT. Yilmaz [28] found similar results by analyzing the relationship between EGT and engine operational parameters at two different power settings, including maximum continuous and take-off, in the CFM56-7B turbofan engine. Hence, the EGT is often used to evaluate the health states of aeroengine and determine the maintenance policy [29].

Aeroengine EGT can be divided into take-off EGT and cruise EGT in accordance with different data acquisition stages during flight [30]. Take-off EGT means the exhaust gas temperature in the take-off stage and maximum thrust. The aeroengine can exceed the normal temperature most easily in this stage which can bring the destructive effect to the engine. In the actual process of engineering application, the take-off EGT margin (EGTM) is often used instead of the take-off EGT parameter to performance analysis. EGTM is the deviation between the actual value and EGT red line value when plane takes off with full power at sea level pressure, inflection point temperature conditions. Among them, EGT red line value refers to the allowed maximum EGT that is given by the manufacturers. The calculation formula of EGTM is defined as

$$
\operatorname{EGTM}\left({ }^{\circ} \mathrm{C}\right)=\mathrm{EGT}_{B}-\mathrm{EGT}_{M},
$$

where subscript $B$ represents a redline value and subscript $M$ represents actual monitoring value.

Figure 1 shows the sequence that denotes the EGTM procured from six CFM56 aeroengines of China Southern [31]. The sampling interval is about a 200-flight cycle, which can be approximately considered continuous equal interval sampling after data preprocessing based on multiple interpolation method.

From Figure 1, it is easy to see that the entire EGTM data sequence has obvious downtrend over time for the six aeroengines. The EGTM can decrease $72^{\circ} \mathrm{C}$ in the 12400 cycle. However, the degradation process of different engines is not quite the same. For a single engine, the changing process of EGTM sequence is a complex nonlinear process. Most of the differences between adjacent sampling points are less than $10^{\circ} \mathrm{C}$ and the maximum reached $20^{\circ} \mathrm{C}$.

Based on the above analysis, aeroengine performance degradation forecast can be solved as EGTM time series forecasting problem. However, it is difficult to establish a precise mathematical model to describe EGTM that can be affected by many uncertain factors. Therefore, the key problem lies in how to establish precise forecast model under 


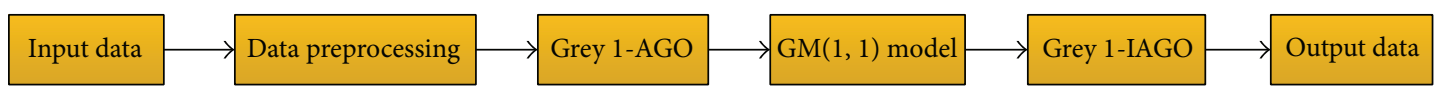

Figure 2: The forecast system based on $\operatorname{GM}(1,1)$ model [32].

incomplete information and discrete small sample data in order to achieve an accurate estimate of the nonlinear EGTM parameters.

\section{EGTM Forecast Modeling Based on GM $(1,1)$ Model}

Based on the temporal variation characteristics of aeroengine EGTM mentioned above, the system of aeroengine performance parameter EGTM can be regarded as a grey dynamic system. This section briefly describes the modeling methodologies about GM $(1,1)$ model and provides an EGTM trend forecasting framework based on GM $(1,1)$ model.

3.1. Modeling Methodologies Based on GM $(1,1)$ Model. In grey systems theory, the most commonly used grey forecast model is GM $(1,1)$ model, which is successfully employed in time series forecast applications with the uncertain problems under discrete data and incomplete information [22].

Generally speaking, forecast based on GM $(1,1)$ model can be regarded as curve fitting analysis in time series [22]. In order to effectively reduce the discreteness of the original time series and reveal the hidden regular pattern in the system development, the first order accumulation generating operation (1-AGO) is used firstly before the first order differential equation is adopted to match the data. Then, whitening equation can be solved by the ordinary least square method to the time response sequences at time $n$. Finally, the first order inverse accumulating generation operation (1IAGO) is employed to establish the GM $(1,1)$ forecast model and obtain the predicted value. The detailed procedure is shown as in Figure 2.

Consider the following nonnegative EGTM time sequence $X^{(0)}$ :

$$
X^{(0)}=\left(x^{(0)}(1), x^{(0)}(2), \ldots, x^{(0)}(n)\right), \quad n \geq 4,
$$

where $n$ is the sample size of the data.

To increase the forecast of the GM $(1,1)$ model, the first order accumulating generation operation (1-AGO) is derived from the original sequence $X^{(0)}$. The 1-AGO is derived as

$$
x^{(1)}(k)=\sum_{i=1}^{k} x^{(0)}(i), \quad k=1,2, \ldots, n .
$$

Then the accumulated sequence $X^{(1)}$ that is obtained using the 1-AGO formation can be expressed as

$$
X^{(1)}=\left(x^{(1)}(1), x^{(1)}(2), \ldots, x^{(1)}(n)\right), \quad n \geq 4 .
$$

The generated mean sequence $Z^{(1)}$ of $X^{(1)}$ is defined as

$$
Z^{(1)}=\left(z^{(1)}(1), z^{(1)}(2), \ldots, z^{(1)}(n)\right),
$$

where $z^{(1)}(k)$ is the mean value of adjacent data; that is,

$$
z^{(1)}(k)=\frac{1}{2} x^{(1)}(k)+\frac{1}{2} x^{(1)}(k-1), \quad k=2,3, \ldots, n .
$$

The grey model GM $(1,1)$ can be expressed by one variable, and the grey difference equation is defined as

$$
x^{(0)}(k)+a z^{(1)}(k)=b .
$$

And its whitening equation is

$$
\frac{d x^{(1)}(t)}{d t}+a x^{(1)}(t)=b,
$$

where coefficients $a$ and $b$ are called developing and grey input coefficients, respectively. By the ordinary least square method, the coefficients $a$ and $b$ can be obtained as

$$
[a, b]^{T}=\left(B^{T} B\right)^{-1} B^{T} Y,
$$

where

$$
\begin{gathered}
Y=\left[x^{(0)}(2), x^{(0)}(3), \ldots, x^{(0)}(n)\right]^{T}, \\
B=\left[\begin{array}{rr}
-z^{(1)}(2) & 1 \\
-z^{(1)}(3) & 1 \\
\cdot & \cdot \\
\cdot & \cdot \\
\cdot & \cdot \\
-z^{(1)}(n) & 1
\end{array}\right] .
\end{gathered}
$$

The solutions $x^{(1)}(t)$ of (8) can be obtained using the ordinary least squares method as follows:

$$
x^{(1)}(t)=\left[x^{(1)}(1)-\frac{b}{a}\right] e^{-a(t-1)}+\frac{b}{a},
$$

where $x^{(1)}(1)=x^{(0)}(1)$.

Hence, the time response sequences of $(8)$ at time $(k+1)$ are

$$
x_{p}^{(1)}(k+1)=\left[x^{(0)}(1)-\frac{b}{a}\right] e^{-a k}+\frac{b}{a} .
$$

To obtain the forecast value of the primitive data at time $(k+1)$, the first order inverse accumulating generation operation (1-IAGO) is employed to establish the following grey model:

$$
x_{p}^{(0)}(k+1)=\left[x^{(0)}(1)-\frac{b}{a}\right] e^{-a k}\left(1-e^{a}\right) .
$$

And the predicted value of the primitive data at time $(k+$ $H)$ is

$$
x_{p}^{(0)}(k+H)=\left[x^{(0)}(1)-\frac{b}{a}\right] e^{-a(k+H-1)}\left(1-e^{a}\right) .
$$




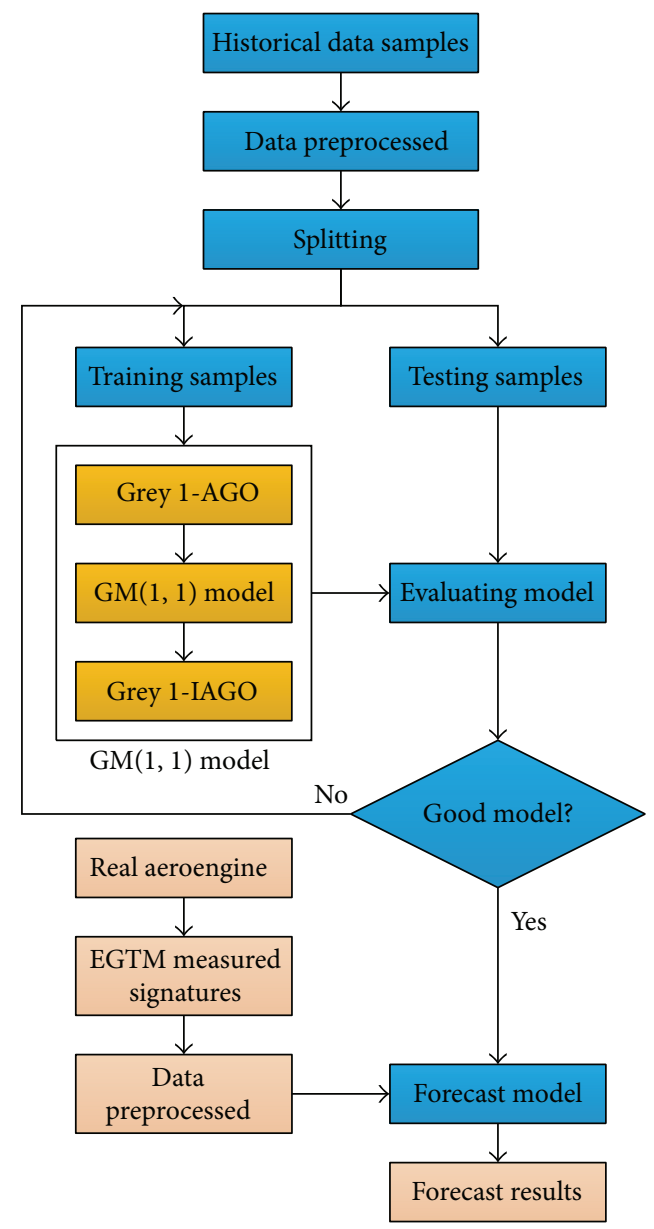

FIGURE 3: Flowchart of EGTM forecast based on GM $(1,1)$ model approach.

Compared with the statistical models, GM $(1,1)$ model need not find the statistics features of original series. So GM $(1,1)$ model gets rid of the shadow of large-sample statistics in terms of information availability degree [24]. Besides, only two coefficients are required to be identified in (8), which means that the number of data sample used in GM $(1,1)$ model is rather small. In other words, GM $(1,1)$ model can realize the forecast by using only sample data sequence and is often used as a short term forecast. This is the biggest advantage over the artificial intelligence method.

\subsection{EGTM Forecast Framework Based on GM $(1,1)$ Model.} According to the above method, a GM $(1,1)$ model based approach for aeroengine performance degradation forecast using EGTM signatures is illustrated in Figure 3. The following shows the details of the forecast process.

Step 1. Generated sufficient EGTM samples from the historical database and the essential preprocessing upon EGTM data are carried out before data analysis, such as supplementary data, eliminating noise and outliers. After that, the samples can be divided into training samples and testing samples.
Step 2. The EGTM forecast model based on GM $(1,1)$ method is established by using the training samples.

Step 3. The testing samples are used to verify the forecast performance of the GM $(1,1)$ model. Step 2 will be repeated if the forecast model accuracies do not meet the requirements.

Step 4. Apply the GM $(1,1)$ model that meets the accurate requirement to EGTM measured signatures obtained from real aeroengine to forecast.

\section{EGTM Forecast Modeling Based on GM (1, 1)-Markov Chain Model}

As a first order single variable grey model, GM $(1,1)$ model provides an excellent approach to forecast uncertain systems [33-37]. However, the forecast accuracy of GM $(1,1)$ model for EGTM series with large random fluctuations is lower, which cannot satisfy the engineering requirement. In order to enhance the forecast performance, Markov chain model is integrated into GM $(1,1)$ model to extract the random fluctuation of experimental data and solve the influence of random fluctuation data on forecast accuracy. The improved model is defined as GM $(1,1)$ Markov chain model.

A GM $(1,1)$ Markov chain based approach for aeroengine performance degradation forecast using EGTM signatures is illustrated in Figure 4. To achieve the aim of this study, the forecast has two stages. The original data are modeled by the GM $(1,1)$ model firstly. Then the residual errors between the fitting values and the actual values for all previous time steps can be obtained. After that, the transition behavior of those residual errors by Markov transition matrices is established and the possible correction for the forecast value can be made from those Markov matrices. The following shows the details of the forecast process.

4.1. Residual Errors. According to the forecast values $x_{p}^{(0)}(k)$ obtained from GM $(1,1)$ model by (13) and the real values $x^{(0)}(k)$, the residual errors series can be obtained as

$$
\varepsilon(k)=x^{(0)}(k)-x_{p}^{(0)}(k),
$$

where $k$ respects the time step.

4.2. Division State. The real values of $x^{(0)}(k)$ are distributed in the region of the forecast value $x_{p}^{(0)}(k)$ which may be divided into a convenient number of contiguous intervals. When $x^{(0)}(k)$ falls in interval $i$, one of $n$ such intervals, it may be regarded as corresponding to a state $Q_{i}$ which can be denoted as follows:

$$
\begin{gathered}
Q_{i}=\left[Q_{1 i}, Q_{2 i}\right], \quad i=1,2, \ldots, n, \\
Q_{1 i}=x_{p}^{(0)}(k)+A_{i}, \quad Q_{2 i}=x_{p}^{(0)}(k)+B_{i},
\end{gathered}
$$

where $Q_{1 i}$ and $Q_{2 i}$ are the lower boundary and upper boundary of the $Q_{i}$ state. $A_{i}$ and $B_{i}$ are the deviation between 


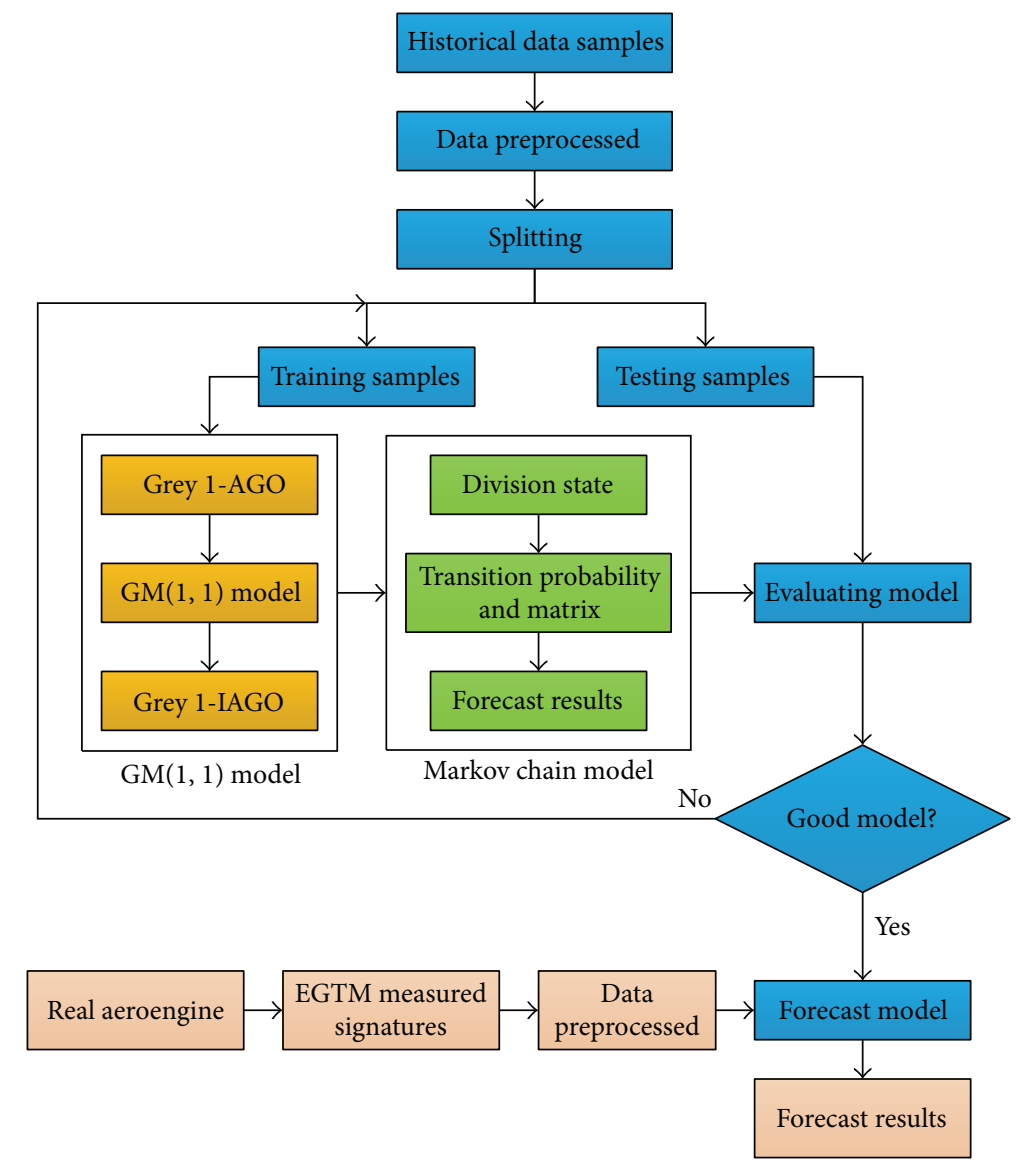

FIGURE 4: Flowchart of EGTM forecast based on GM $(1,1)$ Markov chain approach.

$Q_{1 i}$ and $Q_{2 i}$ with forecast value $x_{p}^{(0)}(k)$, respectively. Hence, $Q_{1 i}$ and $Q_{2 i}$ can reflect the dynamic characteristics of the error residual series.

4.3. Transition Probability and Matrix. Let the state space of a Markov chain $\left\{x_{m}\right\}$ be $I$, the current state be $i$, and the next state be $j$; then, the transition probability is written as

$$
P_{i j}=P\left\{x_{m+1}=j\left|x_{m}=i\right|\right\}, \quad(i, j) \in I,
$$

where the $P_{i j}$ is independent of $m$.

The matrix $P$, formed by placing $P_{i j}$ in row $i$ and column $j$, for all $i$ and $j$, is called the transition probability matrix or chain matrix. Note that the elements of the matrix $P$ satisfy the following two properties:

$$
\begin{aligned}
& P_{i j} \geq 0, \quad \forall i, j \in I, \\
& \sum_{j \in I} P_{i j}=1, \quad \forall i \in I .
\end{aligned}
$$

The transition probability of state is written as

$$
P_{i j}^{(r)}=\frac{M_{i j}^{(r)}}{M_{i}}, \quad i, j=1,2, \ldots, n,
$$

where $P_{i j}^{(r)}$ is the probability of transition from state $i$ to $j$ by $r$ steps. $M_{i j}^{(r)}$ is the transition times from state $i$ to $j$ by $r$ steps and $M_{i}$ is the number of data belonging to the $i$ th state. Because the transition for the last $r$ entries of the series is indefinable, $M_{i}$ should be counted by the first as $l-r$ entries. $l$ is the quantity of entries of the original series. Then, the transition probability matrix of state can be written as

$$
P^{(r)}=\left[\begin{array}{cccc}
P_{11}^{(r)} & P_{12}^{(r)} & \cdots & P_{1 n}^{(r)} \\
P_{21}^{(r)} & P_{22}^{(r)} & \cdots & P_{2 n}^{(r)} \\
\vdots & \vdots & & \vdots \\
P_{n 1}^{(r)} & P_{n 2}^{(r)} & \cdots & P_{n n}^{(r)}
\end{array}\right] .
$$

The transition probability matrix of states $P^{(r)}$ reflects the transition rules of the system. The transition probability of states $P_{i j}^{(r)}$ reflects the probability of transition from initial state $i$ to probable state $j$ by $r$ steps. It is the foundation of forecast by the Markov probability matrix.

Generally speaking, consider $r=1$ and the maximum transition step is 1 . Then, $P^{(1)}$ can be obtained. If the forecast original data is located in the $Q_{i}$ state, the state of next step is determined by the $i$ th row vector of transition probability states $P_{i j}^{(1)}$. If $\max P_{i j}^{(1)}=P_{i 1}^{(1)}$, the state of next step is $Q_{1}$. 
When the state of next step cannot be determined by 1-step transition probability, the 2 -step transition probability will be selected.

4.4. Obtaining the Forecast Value. When the possibility of a certain state of the next step is determined by the probabilities in $k-1$ row vectors, $Q_{1 i}$ and $Q_{2 i}$ can also be obtained. The median in $\left[Q_{1 i}, Q_{2 i}\right]$ is selected as the forecast value, so forecast value of original data sequence is obtained according to the above explanation.

$$
\widehat{x}_{p}^{(0)}(k)=x_{p}^{(0)}(k)+\left(\frac{A_{i}+B_{i}}{2}\right) .
$$

The main assumption in a Markov chain model is that knowledge of the current state occupied by the process can be sufficient to describe the future probabilistic behavior of the process. Another unique property of this Markov chain model is the existence of a steady state matrix.

\section{Experimental Study}

In this section, the forecast approach based on $\operatorname{GM}(1,1)$ Markov chain introduced in this paper will be applied to EGTM forecast of CFM56 aeroengine to demonstrate the potential capability of the new approach. The comparisons between the EGTM forecast capabilities using the $\operatorname{GM}(1,1)$ model, GM $(1,1)$ Markov chain model, and other traditional methods are adopted. Four performance measures are used to examine the forecast accuracy of forecast models in this paper. The relative percentage error (RPE), mean square error (MSE), absolute mean error (AME), and absolute mean percentage error (AMPE) are calculated using the following functions, respectively:

$$
\begin{aligned}
\mathrm{RPE} & =\frac{|\varepsilon(k)|}{x^{(0)}(k)} \times 100 \%, \\
\mathrm{MSE} & =\frac{1}{n} \sum_{k=1}^{n} \varepsilon(k)^{2}, \\
\mathrm{AME} & =\frac{1}{n} \sum_{k=1}^{n}|\varepsilon(k)|, \\
\mathrm{AMPE} & =\frac{1}{n} \sum_{k=1}^{n} \frac{|\varepsilon(k)|}{x^{(0)}(k)} \times 100 \% .
\end{aligned}
$$

5.1. Sample Data. For this study, the investigators gathered data samples from CFM56 aeroengine of China Southern, which have been described in Figure 1. In order to highlight the forecast performance of the forecast methods under incomplete information and discrete small sample data, only 45 samples are taken to construct a time series $\left\{\mathrm{EGTM}_{m}\right\}(m=1,2, \ldots, 45)$ from aeroengine 2 . Figure 5 shows the distribution of training and testing sample data. As the training sample, the first 20 sample data are first used to establish forecast model. Then the next 25 sample data are used to test the effectiveness of forecast model.

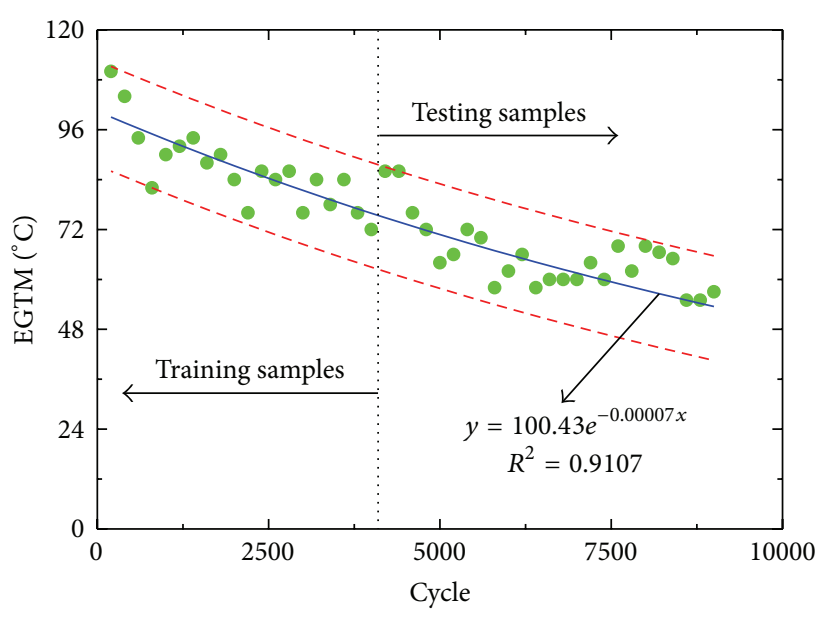

- Aeroengine 2

FIGURE 5: Sample sets partition for EGTM forecast.

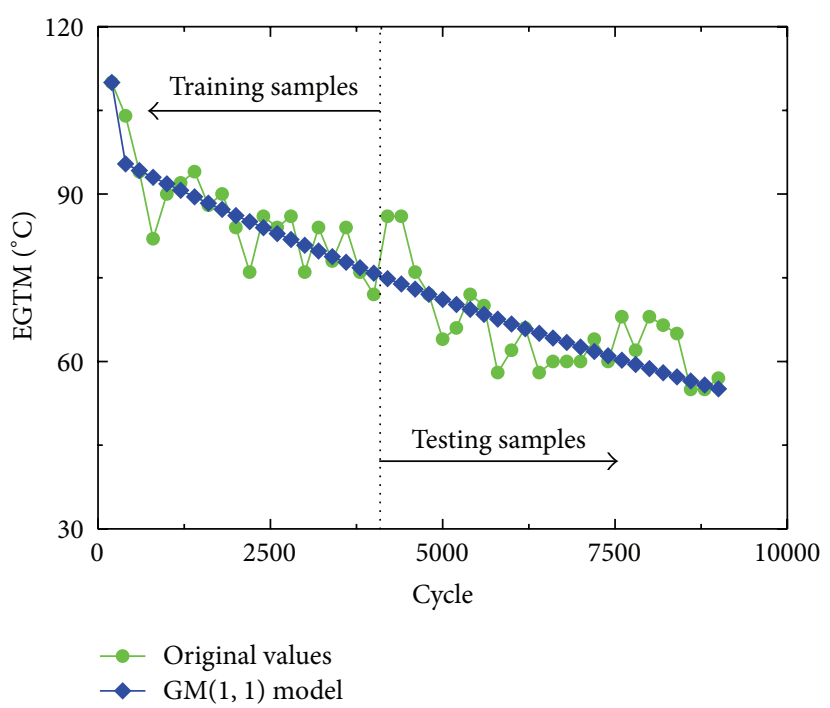

Figure 6: Forecast values of EGTM based on GM $(1,1)$ model.

5.2. Forecast Results Based on GM $(1,1)$ Model. After the generation of samples, GM $(1,1)$ model is established by (13) to forecast the trend from the generated training samples. Specific modeling steps and methods have been given in Figure 3.

The forecast results of EGTM based on GM $(1,1)$ model and the original series are plotted in Figure 6. From Figure 6, it is clear that the trend of EGTM can be forecasted based on GM $(1,1)$ model. However, the forecast accuracies of GM (1, 1) model are unstable at some points. And because the accumulated sequence obtained using the 1-AGO formation is monotonically increasing, which is seen in Figure $7, \operatorname{GM}(1,1)$ model cannot extract random fluctuations of EGTM.

In order to compare other models, the linear regression model $y=-0.0064 x+99.968$, nonlinear regression model $y=100.19 \exp (-0.00007 x)$, ARIMA $(1,0,0)$ model, and Radial Basis Function (RBF) Neural Networks are also 
TABLE 1: The comparison results of GM $(1,1)$ model and GM $(1,1)$ Markov chain model.

\begin{tabular}{|c|c|c|c|c|c|c|}
\hline \multirow{2}{*}{ Cycle } & \multicolumn{6}{|c|}{ Forecast model } \\
\hline & Original values & Linear model & Nonlinear model & ARIMA model & RBF model & GM $(1,1)$ model \\
\hline 4200 & 86 & 73.088 & 74.66925168 & 76.24820454 & 83.893778 & 74.850179 \\
\hline 4400 & 86 & 71.808 & 73.63116571 & 85.04659158 & 75.345291 & 73.899849 \\
\hline 4600 & 76 & 70.528 & 72.60751169 & 85.09212012 & 66.440678 & 72.961583 \\
\hline 4800 & 72 & 69.248 & 71.59808897 & 79.58775994 & 77.213138 & 72.035231 \\
\hline 5000 & 64 & 67.968 & 70.60269971 & 69.85924741 & 75.907728 & 71.12064 \\
\hline 5200 & 66 & 66.688 & 69.6211488 & 69.85924741 & 51.433499 & 70.217661 \\
\hline 5400 & 72 & 65.408 & 68.65324386 & 70.37022346 & 77.51105 & 69.326147 \\
\hline 5600 & 70 & 64.128 & 67.69879518 & 74.57638112 & 68.512595 & 68.445951 \\
\hline 5800 & 58 & 62.848 & 66.75761567 & 72.84613016 & 52.139635 & 67.576931 \\
\hline 6000 & 62 & 61.568 & 65.82952088 & 62.6255194 & 75.584126 & 66.718945 \\
\hline 6200 & 66 & 60.288 & 64.91432888 & 65.48119394 & 63.496734 & 65.871852 \\
\hline 6400 & 58 & 59.008 & 64.01186029 & 68.51302315 & 64.808649 & 65.035514 \\
\hline 6600 & 60 & 57.728 & 63.12193824 & 61.40259349 & 54.990815 & 64.209794 \\
\hline 6800 & 60 & 56.448 & 62.24438829 & 62.78391435 & 60.518705 & 63.394558 \\
\hline 7000 & 60 & 55.168 & 61.37903843 & 62.49368451 & 62.428861 & 62.589673 \\
\hline 7200 & 64 & 53.888 & 60.52571907 & 62.25825584 & 61.24122 & 61.795007 \\
\hline 7400 & 60 & 52.608 & 59.68426294 & 65.60171099 & 57.527047 & 61.01043 \\
\hline 7600 & 68 & 51.328 & 58.85450511 & 62.02015608 & 58.92226 & 60.235815 \\
\hline 7800 & 62 & 50.048 & 58.03628296 & 68.8277359 & 53.706033 & 59.471034 \\
\hline 8000 & 68 & 48.768 & 57.22943611 & 63.72946594 & 60.035962 & 58.715964 \\
\hline 8200 & 66.5 & 47.488 & 56.4338064 & 68.75093548 & 60.237787 & 57.97048 \\
\hline 8400 & 65 & 46.208 & 55.64923791 & 67.47264457 & 67.031388 & 57.234461 \\
\hline 8600 & 55 & 44.928 & 54.87557684 & 66.17459205 & 53.436919 & 56.507787 \\
\hline 8800 & 55 & 43.648 & 54.11267156 & 57.5174732 & 55.602918 & 55.79034 \\
\hline 9000 & 57 & 42.368 & 53.36037254 & 57.31506291 & 58.04245 & 55.082001 \\
\hline
\end{tabular}

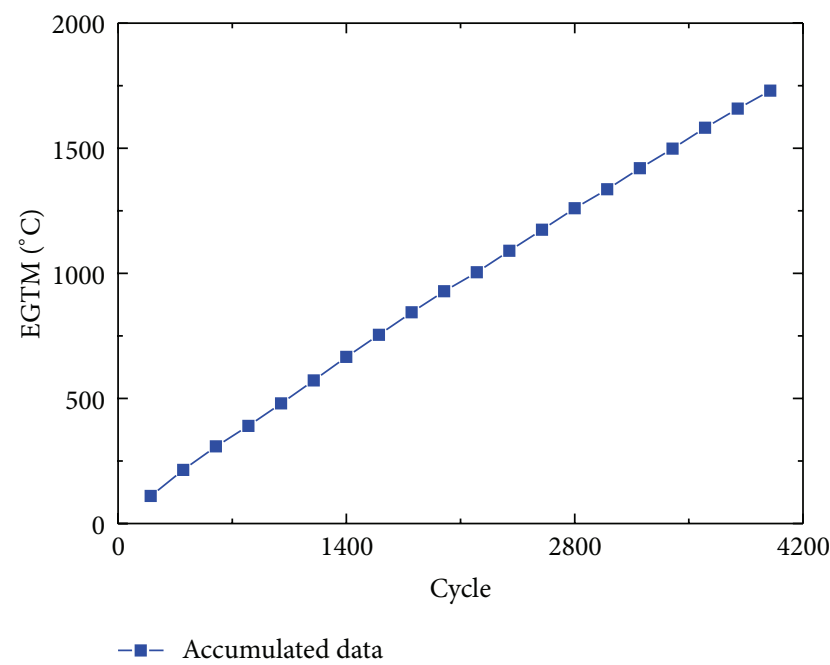

FIGURE 7: The accumulated data of EGTM using the 1-AGO formation.

employed to make the same forecasts. The comparative results are listed in Table 1 . We can see that the forecast performance based on nonlinear model is closely approximate to the forecast performance based on $\operatorname{GM}(1,1)$ model. Besides, GM $(1,1)$ model is better than linear model or RBF model that often requires a large amount of training samples to get a higher forecast precision. Furthermore, the differences between GM $(1,1)$ model and ARIMA $(1,0,0)$ model are that $\operatorname{GM}(1,1)$ model does not have a requirement to the distribution characteristics of training samples while ARIMA $(1,0,0)$ model not only requires a large amount of training samples, but also requires the law for the distribution of training samples. Considering the above factors, $\operatorname{GM}(1,1)$ model is more suitable for EGTM forecast, especially for small sample data.

\subsection{Forecast Results Based on GM $(1,1)$ Markov Chain Model}

5.3.1. Division State. In the previous literature, there is no unified standard to determine number of state and the boundary of the $Q_{i}$ state, and state division is performed on experience, which is called the hard division approach, which might result in the weakness of forecast accuracy and algorithm application, due to the fact that the different divisions would be made by different persons. Generally speaking, the value ranges of $n$ are $3-6$, and they ensure that each state interval has data. 


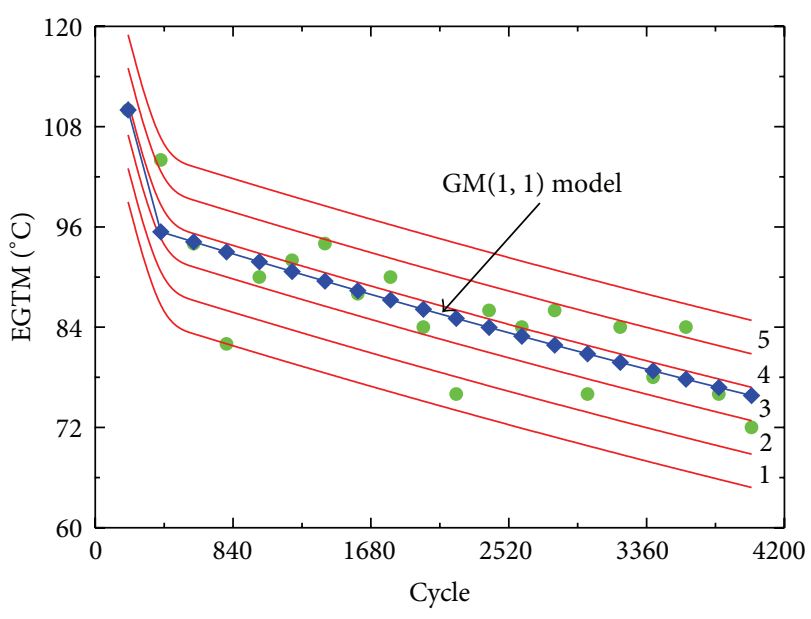

- Aeroengine 2

FIGURE 8: State division for the time series of EGTM.

According to the absolute error series $\varepsilon(k)$, the states are partitioned by establishing five contiguous intervals for Markov-chain forecast model. Figure 8 shows the results of state division. The blue curve shows the obtained trend curve by GM $(1,1)$ model and the red curves show the state lines.

5.3.2. Transition Probability and Matrix. From Figure 8, it can be seen that the number of samples in each state is as follows: $M_{1}(1)=2, M_{2}(1)=1, M_{3}(1)=7, M_{4}(1)=7, M_{2}(1)=2$. Then one-step transition probability for every state can be calculated by using (19) and (20). Consider the following:

$$
P^{(1)}=\left[\begin{array}{ccccc}
0 & 0 & \frac{1}{2} & \frac{1}{2} & 0 \\
0 & 0 & 0 & 1 & 0 \\
\frac{2}{7} & \frac{1}{7} & 0 & \frac{2}{7} & \frac{2}{7} \\
0 & \frac{1}{7} & \frac{3}{7} & \frac{3}{7} & 0 \\
0 & 0 & 1 & 0 & 0
\end{array}\right]
$$

5.3.3. Forecast Values and Analysis. When the possibility of a certain state of the next step is determined according to 1step probability transition matrices $P^{(1)}, Q_{1 i}$ and $Q_{2 i}$ can be obtained. The forecast values can be obtained through the same computations. The forecast values by GM $(1,1)$ model and GM $(1,1)$ Markov chain model are shown in Figure 9. What is not worthy is that the dynamic change characteristics of EGTM are reflected by calculating the state transfer probability. Figures 10, 11, and 12 show the RPE comparative analysis of different forecast models. From the values of RPE comparison, we find that the forecast accuracies of GM (1, 1) Markov chain model are significantly higher than other models.

The comparison of forecast accuracy of the different models is listed in Table 2. From Table 2, it is easy to see

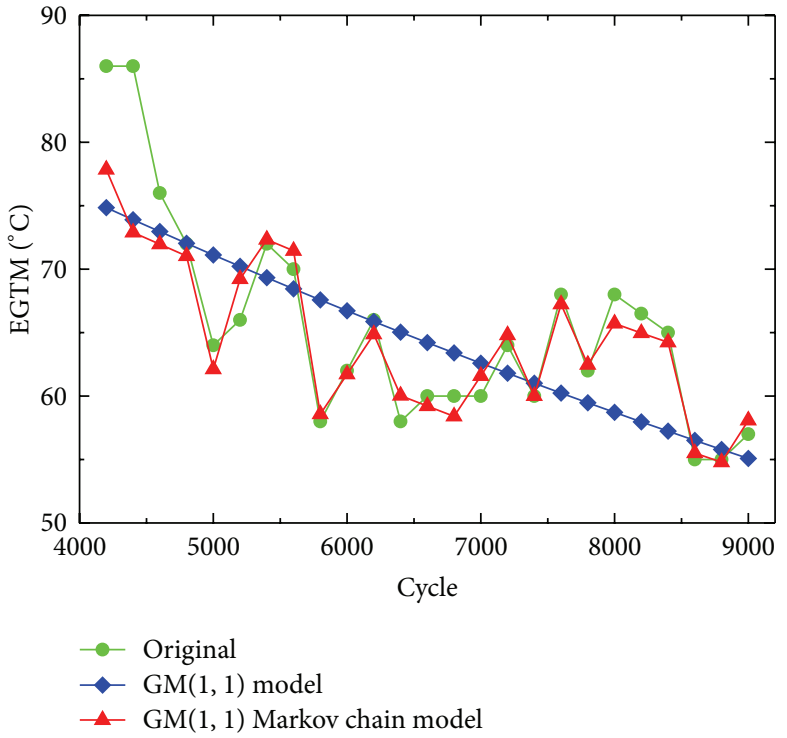

FIGURE 9: : The forecast values by GM $(1,1)$ model and GM $(1,1)$ Markov chain model for EGTM.

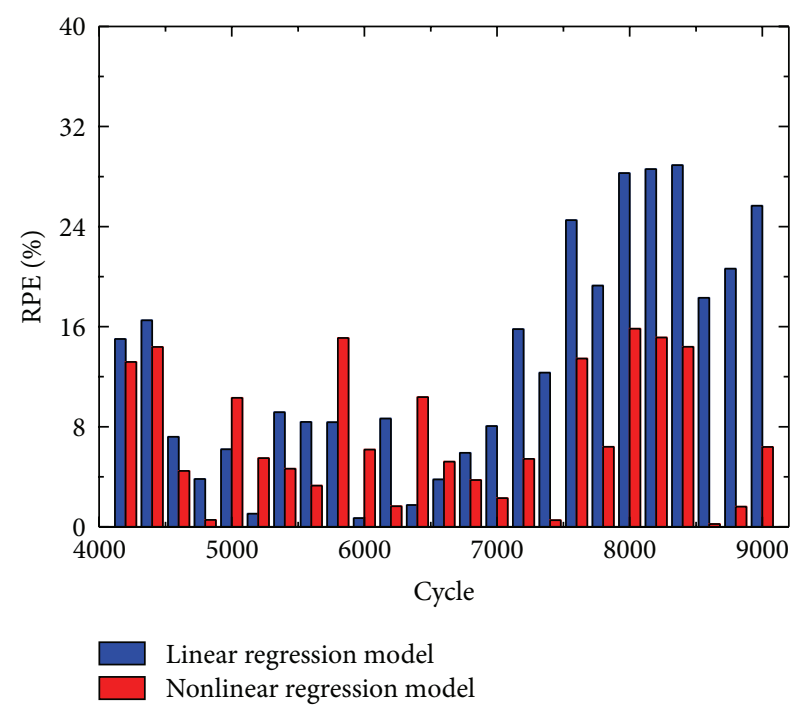

FIGURE 10: The forecast RPE by linear regression model and nonlinear regression model for EGTM.

TABLE 2: The comparative analysis results of different models.

\begin{tabular}{lccc}
\hline \multirow{2}{*}{ Models } & \multicolumn{3}{c}{ Evaluating standards } \\
& MSE & AME & AMPE/\% \\
\hline Linear model & 108.1934 & 8.572960000 & 13.07414 \\
Nonlinear model & 37.67832 & 4.861358116 & 7.209246 \\
ARIMA model & 37.36459 & 4.785827391 & 7.447413 \\
RBF model & 48.05668 & 5.59155023 & 8.443975 \\
GM $(1,1)$ model & 34.2345 & 4.673887228 & 6.982612 \\
GM $(1,1)$ Markov chain model & 11.75861 & 1.981937814 & 2.727393 \\
\hline
\end{tabular}

that the forecast performance of GM $(1,1)$ Markov chain model outperforms other models. When all of the factors 


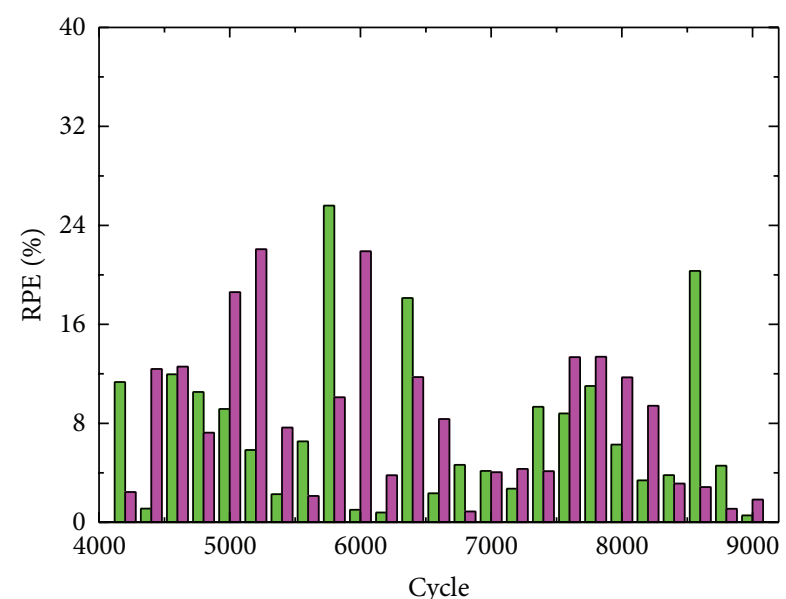

ARMIA model

RBF model

Figure 11: The forecast values by ARMIA $(1,0,0)$ model and RBF model for EGTM.

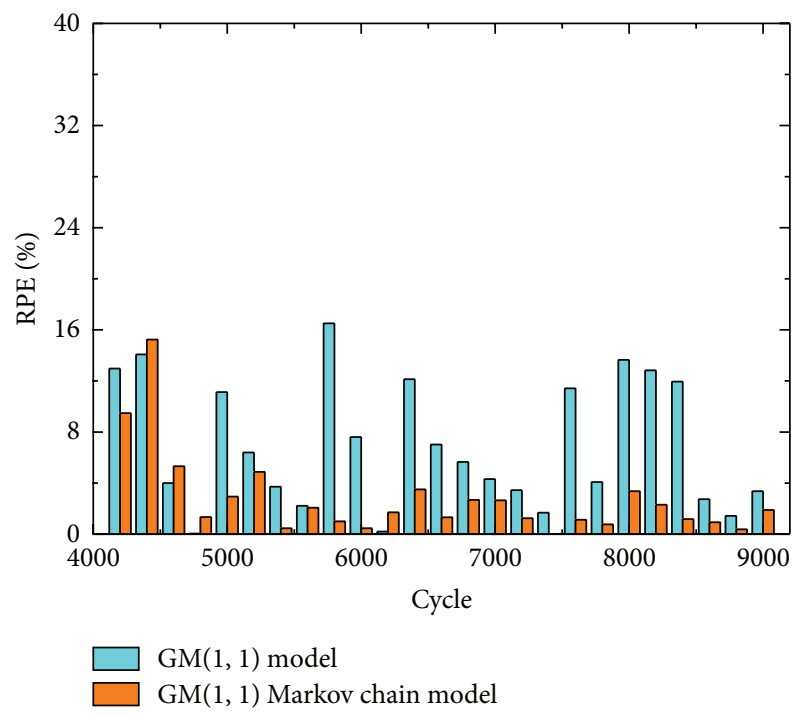

Figure 12: The forecast values by GM $(1,1)$ model and GM $(1,1)$ Markov chain model for EGTM.

are considered together, the modeling processes of GM $(1,1)$ Markov chain model are much easier than ARIMA $(1,0,0)$ model and RBF model. Through the test values, we find that GM $(1,1)$ Markov chain model gives more satisfactory performances in MSE, AME, and AMPE than other models. The AMPE of GM $(1,1)$ Markov chain model is only $2.727393 \%$, which can meet the demand of engineering application. The results validate the effectiveness of GM $(1,1)$ Markov chain model.

\section{Conclusions}

According to the characteristics of the aeroengine gas path performance parameters, EGTM is used to realize aeroengine performance degradation forecast. Based on the change law of aeroengine EGTM, EGTM forecast is solved as a grey system forecast problem. However, it is shown that GM (1, 1) model is only able to accurately forecast the trend of EGTM and the forecast accuracy of GM $(1,1)$ model is not satisfactory when the EGTM data show great randomness. In order to enhance the forecast performance of GM $(1,1)$ model, Markov chain model is integrated into GM $(1,1)$ model. The comparison results show that the forecast accuracy of the improved model named GM $(1,1)$ Markov chain model is better than other models, especially for the small samples. GM $(1,1)$ Markov chain model can solve the influence of random fluctuation data on forecast accuracy and achieve an accurate estimate of the nonlinear EGTM.

\section{Conflict of Interests}

The authors declared that there is no conflict of interests regarding the publication of this paper.

\section{Acknowledgment}

The present work is supported by the Fundamental Research Funds for the Central Universities of China (no. HEUCFZ1005).

\section{References}

[1] C. S. Bytington, M. J. Roemer, and T. Galie, "Prognostic enhancements to diagnostic systems for improved conditionbased maintenance," in Proceedings of the IEEE Aerospace Conference, vol. 6, pp. 2815-2824, 2002.

[2] L. Marinai, D. Probert, and R. Singh, "Prospects for aero gasturbine diagnostics: a review," Applied Energy, vol. 79, no. 1, pp. 109-126, 2004.

[3] J. S. Litt, D. L. Simon, and S. Garg, "A survey of intelligent control and health management technologies for aircraft propulsion systems," Tech. Rep. NASA-TM-2005-213622, 2005.

[4] K. J. Melcher, W. A. Maul, and S. Garg, "Propulsion health management system development for affordable and reliable operation of space exploration systems," AIAA 2007-6237, 2007.

[5] Y. G. Li, "Gas turbine performance and health status estimation using adaptive gas path analysis," Journal of Engineering for Gas Turbines and Power, vol. 132, no. 4, Article ID 041701, 9 pages, 2010.

[6] G. Chen, "Forecasting engine performance trend by using structure self-adaptive neural network," Acta Aeronautica et Astronautica Sinica, vol. 28, no. 3, pp. 535-539, 2007.

[7] L. P. Jiang, "Gas path fault diagnosis system of aero-engine based on grey relationship degree," Procedia Engineering, vol. 15, pp. 4774-4779, 2011.

[8] X. H. Wu, D. Wen, R. G. Li, Z. Z. Chen, H. Z. Huang, and Z. Q. Lv, "A model to predict the residual life of aeroengine based upon Support Vector Machine," in Proceedings of the International Conference on Quality, Reliability, Risk, Maintenance, and Safety Engineering (QR2MSE '13), pp. 18801882, Chengdu, China, July 2013.

[9] S. H. Ren and H. F. Zuo, "A combined prediction method for the residual-life of civil aviation engines based on performance 
degradation," Mechanical Science and Technology For Aerospace Engineering, vol. 30, no. 1, pp. 23-29, 2011.

[10] X. Y. Fu, S. S. Zhong, and M. N. Zhang, "An improved method for aeroengine residual life prediction," Journal of Harbin Institute of Technology, vol. 45, no. 5, pp. 51-55, 2013.

[11] H. X. Wang and Y. Jiang, "Performance reliability prediction of complex system based on the condition monitoring information," Mathematical Problems in Engineering, vol. 2013, Article ID 836517, 7 pages, 2013.

[12] Y. S. Shi, Y. Z. Li, and Y. X. Song, "Aeroengine performance parameter forecast based on partial least-squares regression method under condition of small samples," Aircraft Design, vol. 32, no. 6, pp. 47-51, 2012.

[13] X. B. Li, X. L. Cui, and R. L. Lang, "Forecasting method for aeroengine performance parameters," Journal of Beijing University of Aeronautics and Astronautics, vol. 34, no. 3, pp. 253-256, 2008.

[14] S. S. Zhong, D. Lei, and G. Ding, "Convolution sum discrete process neural network and its application in aeroengine exhausted gas temperature prediction," Acta Aeronautica et Astronautica Sinica, vol. 33, no. 3, pp. 438-445, 2012.

[15] S. S. Zhong, S. G. Luan, and G. Ding, "Aeroengine condition monitoring based on process neural networks and aerothermoparameters," Journal of Nanjing University of Science and Technology, vol. 30, no. 5, pp. 533-536, 2006.

[16] G. Ding, M. Q. Xu, and L. G. Hou, "Prediction of aeroengine exhaust gas temperature using process neural network," Journal of Aerospace Power, vol. 24, no. 5, pp. 1035-1039, 2009.

[17] C. X. Zhang and N. Wang, "Aero-engine condition monitoring based on support vector machine," Physics Procedia, vol. 24, part B, pp. 1546-1552, 2012.

[18] X. Y. Fu and S. S. Zhong, "Aeroengine turbine exhaust gas temperature prediction using process support vector machines," in Advances in Neural Networks, vol. 7951, pp. 300-310, Springer, Berlin, Germany, 2013.

[19] M. Ilbas and M. Turkmen, "Estimation of exhaust gas temperature using artificial neural network in turbofan engines," Journal of Thermal Science and Technology, vol. 32, no. 2, pp. 11-18, 2012.

[20] J. J. Flores, M. Graff, and H. Rodriguez, "Evolutive design of ARMA and ANN models for time series forecasting," Renewable Energy, vol. 44, pp. 225-230, 2012.

[21] J. L. Deng, "Control problems of grey systems," Systems \& Control Letters, vol. 1, no. 5, pp. 288-294, 1982.

[22] E. Kayacan, B. Ulutas, and O. Kaynak, "Grey system theorybased models in time series prediction," Expert Systems with Applications, vol. 37, no. 2, pp. 1784-1789, 2010.

[23] Z. F. Wu, L. Guo, and H. F. Zuo, "The wear fault prediction model of aero-engine based on the gray system theory," in Proceedings of the IEEE International Conference on Grey Systems and Intelligent Services (GSIS '07), pp. 528-532, Nanjing, China, November 2007.

[24] G. D. Li, S. Masuda, and M. Nagai, “The prediction model for electrical power system using an improved hybrid optimization model," International Journal of Electrical Power \& Energy Systems, vol. 44, no. 1, pp. 981-987, 2013.

[25] L. Wang, Y. G. Li, M. F. Abdul Ghafir, and A. Swingler, "A Rough Set-based gas turbine fault classification approach using enhanced fault signatures," Proceedings of the Institution of Mechanical Engineers A, vol. 225, no. 8, pp. 1052-1065, 2011.

[26] P. E. P. Hamilton and D. Ha, "Exhaust gas temperature capabilities now in system 1 software," Product Update, vol. 25, no. 1, pp. 88-89, 2005.
[27] Y. X. Song, K. X. Zhang, and Y. S. Shi, "Research on aeroengine performance parameters forecast based on multiple linear regression forecasting method," Journal of Aerospace Power, vol. 24, no. 2, pp. 427-431, 2009.

[28] I. Yilmaz, "Evaluation of the relationship between exhaust gas temperature and operational parameters in CFM56-7B engines," Proceedings of the Institution of Mechanical Engineers G, vol. 223, no. 4, pp. 433-440, 2009.

[29] E. M. He and L. T. Song, "Analysis of EGT and measures to increase the EGT margin," Aviation Engineering \& Maintenance, vol. 6, pp. 20-21, 1999.

[30] M. Bengtsson, "Condition based maintenance system technology-where is development heading?" in Proceedings of the 17th European Maintenance Congress, Barcelona, Spain, May 2004.

[31] Y. Z. Zhao, Research on methods of life on wing prediction for civil aeroengine [M.S. thesis], Nanjing University of Aeronautics and Astronautics, Nanjing, China, 2010.

[32] J. L. Deng, "Introduction to grey system theory," The Journal of Grey System, vol. 1, no. 1, pp. 1-24, 1989.

[33] Y. He and Y. D. Bao, "Grey-Markov forecasting model and its application," System Engineering: Theories \& Practice, vol. 9, no. 4, pp. 59-63, 1992.

[34] M. Huang, Y. He, and H. Cen, "Predictive analysis on electricpower supply and demand in China," Renewable Energy, vol. 32, no. 7, pp. 1165-1174, 2007.

[35] U. Kumar and V. K. Jain, “Time series models (Grey-Markov, Grey Model with rolling mechanism and singular spectrum analysis) to forecast energy consumption in India," Energy, vol. 35, no. 4, pp. 1709-1716, 2010.

[36] G. D. Li, D. Yamaguchi, and M. Nagai, "A GM(1,1)-Markov chain combined model with an application to predict the number of Chinese international airlines," Technological Forecasting and Social Change, vol. 74, no. 8, pp. 1465-1481, 2007.

[37] Y. T. Hsu, M. C. Liu, J. Yeh, and H. F. Hung, "Forecasting the turning time of stock market based on Markov-Fourier grey model," Expert Systems with Applications, vol. 36, no. 4, pp. 8597-8603, 2009. 


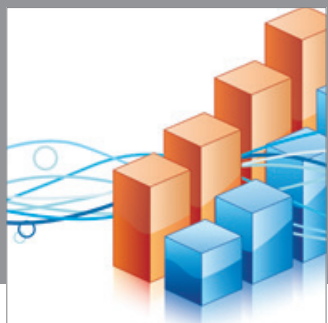

Advances in

Operations Research

mansans

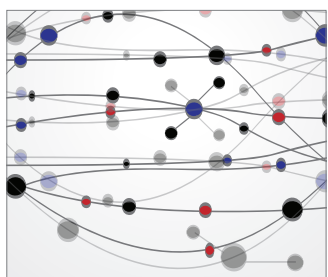

The Scientific World Journal
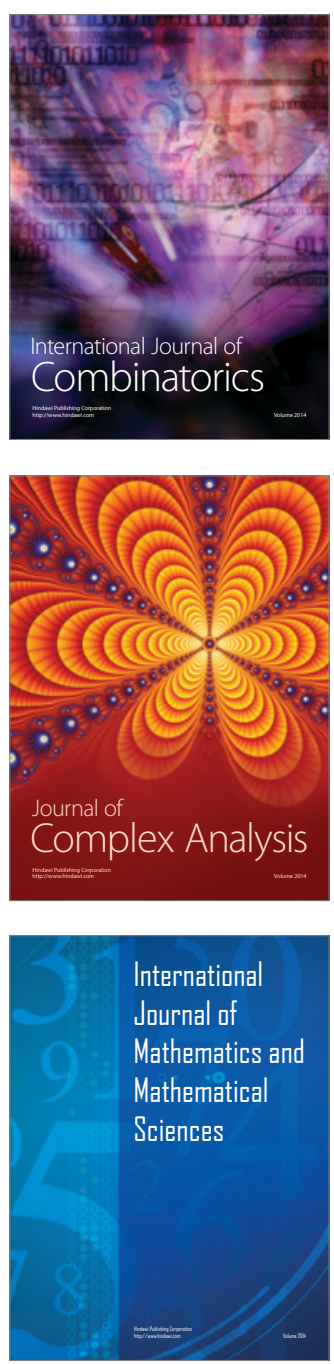
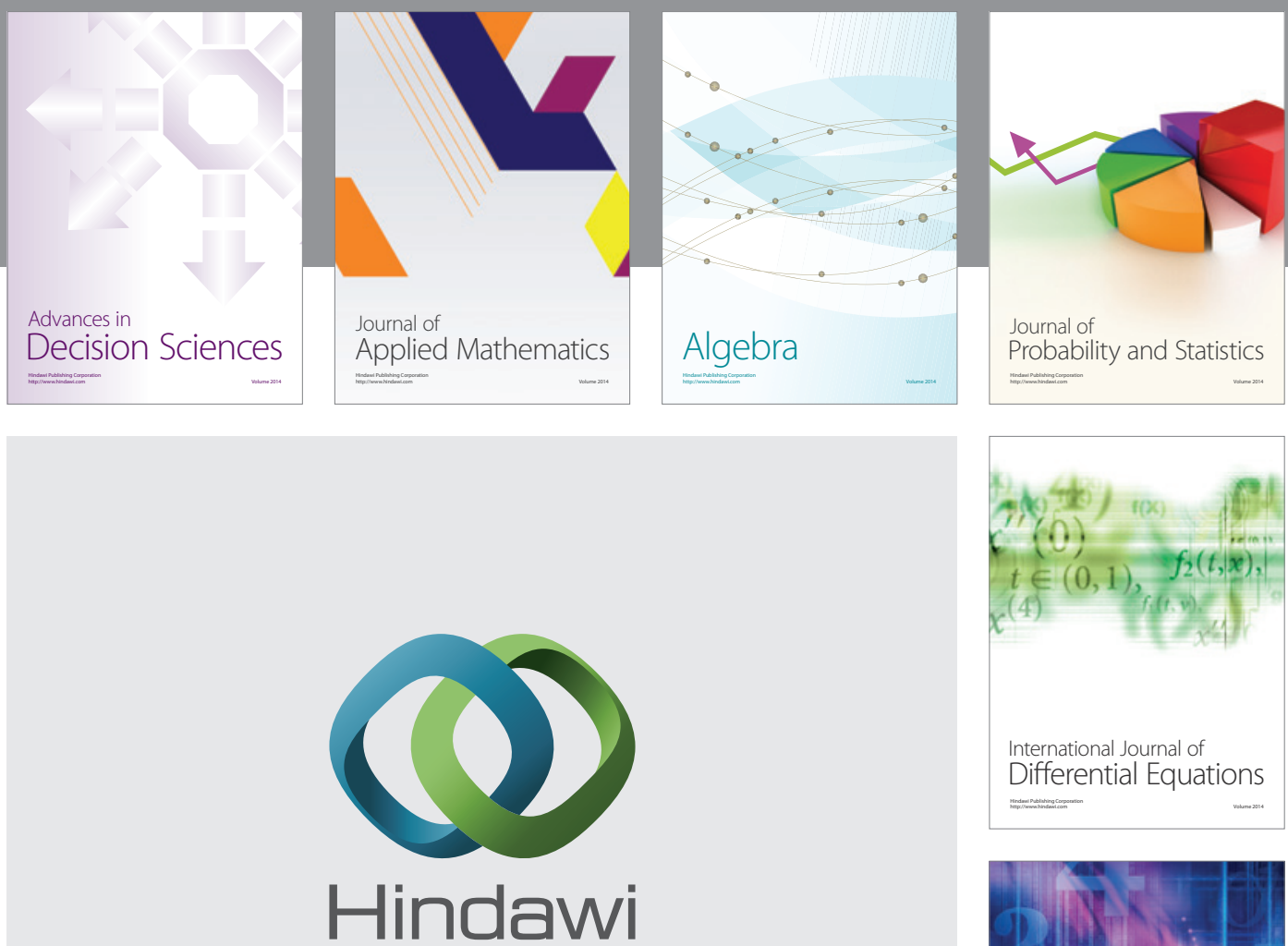

Submit your manuscripts at http://www.hindawi.com
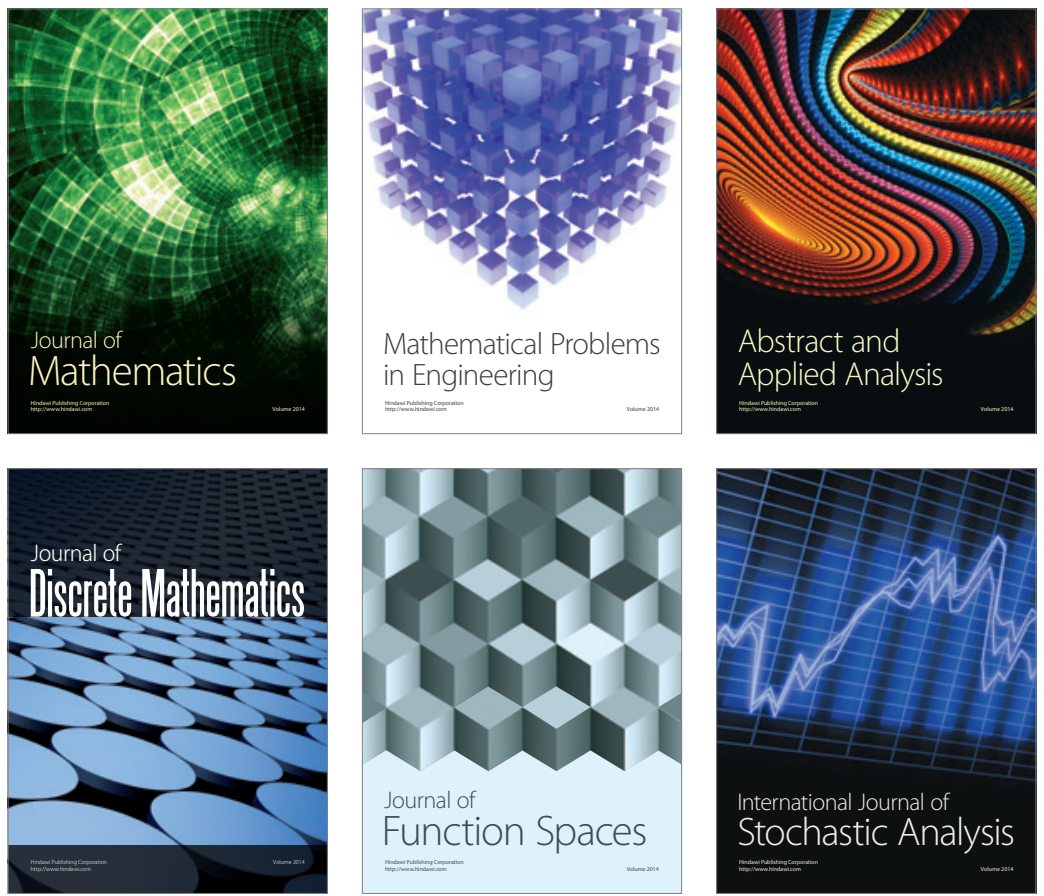

Journal of

Function Spaces

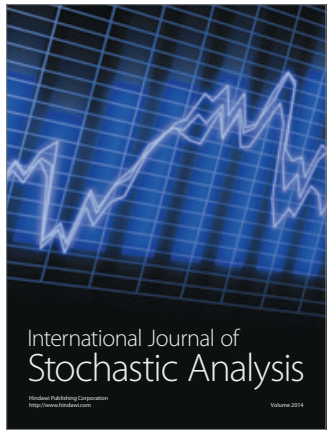

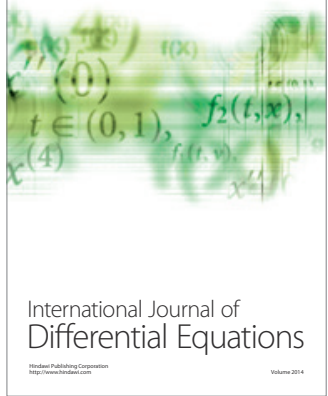
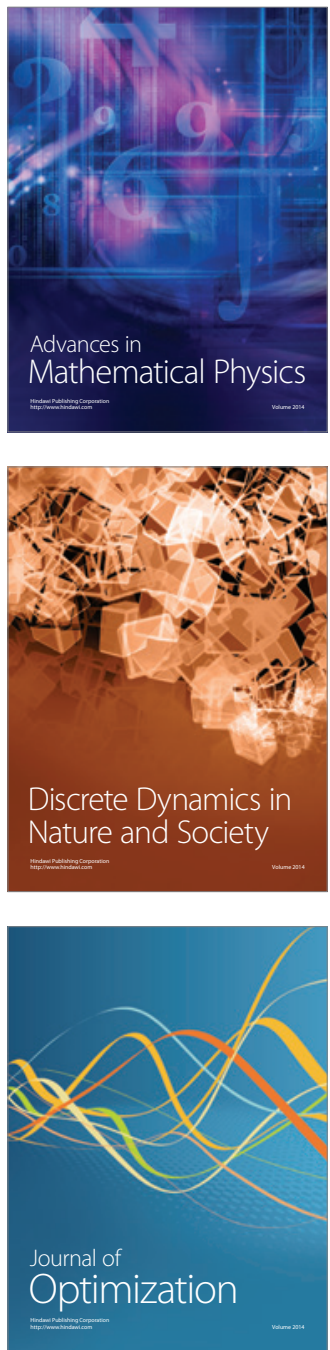